\title{
A Terbium Sensitized Luminescence Method for the Assay of Flubiprofen in Pharma- ceutical Formulations
}

\author{
Salma M.Z. Al-Kindy*, Arwa Al-Ghazali, Fakhr Eldin O. Suliman and Haidar A.J. Al-Lawati \\ Department of Chemistry, College of Science, Sultan Qaboos University, P.O. Box: 36, PC 123, \\ Al-Khod, Muscat, Sultanate of Oman. *Email: alkindy@squ.edu.om.
}

\begin{abstract}
A sensitive time-resolved luminescence method for the determination of flubiprofen (FLP) in methanol and in aqueous solution is described. The method is based on the luminescence sensitization of terbium $\left(\mathrm{Tb}^{3+}\right)$ by the formation of a ternary complex with FLP in the presence of 4,7-diphenyl-1,10-phenanthroline (DPP) as co-ligand, and Tween-20 as surfactant. The signal for Tb-FLP-DPP was monitored at $\lambda_{\mathrm{ex}}=285 \mathrm{~nm}$ and $\lambda_{\mathrm{em}}=552 \mathrm{~nm}$. Optimum conditions for the formation of the complex in an aqueous system were TRIS buffer, $\mathrm{pH} 8.0$, DPP $\left(2.5 \times 10^{-7} \mathrm{M}\right)$, Tween-20 (0.30\%) and $4 \times 10^{-5} \mathrm{~mol} \mathrm{~L}^{-1}$ of $\mathrm{Tb}^{3+}$ which allowed the determination of $20-1000 \mathrm{ng} \mathrm{mL}^{-1}$ of FLP with a limit of detection (LOD) of $10 \mathrm{ng} \mathrm{mL}^{-1}$. The relative standard deviations of the method ranged between 0.6 and $1.4 \%$ indicating excellent reproducibility of the method. The proposed method was successfully applied for the assays of FLP in pharmaceutical formulations and spiked tap water samples with average recoveries of $87 \%-95 \%$.
\end{abstract}

Keywords: Flurbiprofen; Terbium; Sensitized luminescence; Drug formulation.

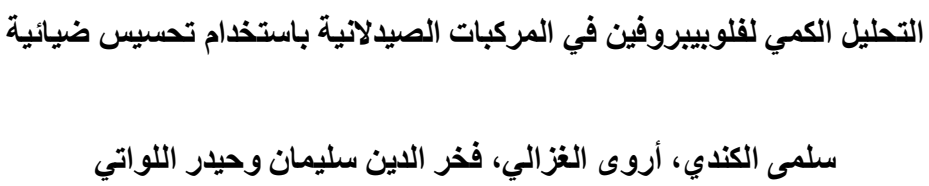

ملخص: نم وصف طريقة حساسة قائمة على التحليل الزمني للضيائية لتحديد الفلوبييروفين (FLP) في الميثنانول و المحاليل المائية، وتعتمد الطريقة على

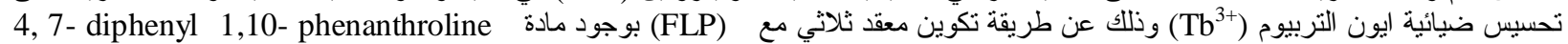

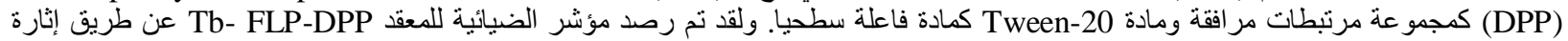

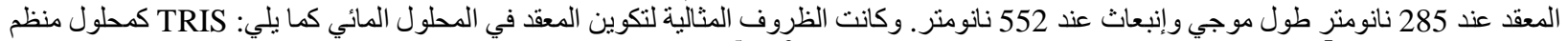

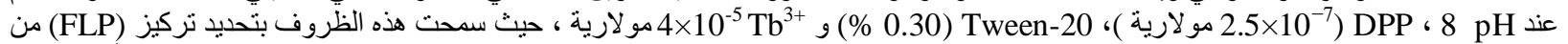

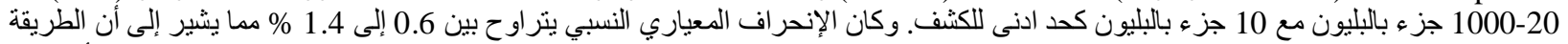

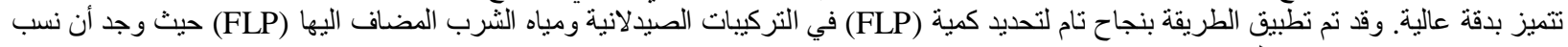

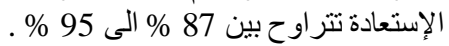

\section{Introduction}

$$
\text { مفتاح الكلمات: ايون التربيوم فلوبييروفين، التربيوم، تحسيس الضيائية والمركبات الصيدلانية. }
$$

F lubiprofen (FLP) [( \pm )-2-(2-fluoro-4-biphenylyl) propionic acid]) is a non-steroidal anti-inflammatory drug (NSAID), effectively used in the treatment of rheumatoid arthritis. It belongs to the profen derivatives, a class of phenyl propanoic acids that has anti-inflammatory effects in addition to antipyretic and analgesic activity. The antiinflammatory effect of FLP is assigned to the inhibition of COX (Cyclooxygenase) and, in particular, COX-2 activity that is known to block the conversion of arachidonic acid to prostaglandin, which is an indication of inflammation [1].

There is evidence that numerous NSAIDs, including FLP, may help to reduce the severity of Alzheimer's disease (AD). The beneficial effect is attributed to the reduction of the inflammation response of the AD brain which minimizes brain lesion and protects brain cells against damage, thus resulting in an improved memory and cognitive function [2]. 


\section{A TERBIUM SENSITIZED LUMINESCENCE METHOD}

FLP demonstrates comparable efficacy to other NSAIDs such as aspirin, ibuprofen, and naproxen in the treatment of rheumatoid arthritis. On the other hand, however, NSAIDs have some side effects; they may cause an increased risk of serious cardiovascular thrombotic events, myocardial infarction, stroke and serious gastrointestinal adverse events including bleeding, ulceration, and perforation of the stomach or intestines which can be fatal [3].

Simple, accurate, precise and robust analytical techniques that could be applied directly and easily to pharmaceutical preparations are necessary, especially with the continuous production of new drugs every year and the increasing use of these drugs. Several analytical techniques have been developed for the determination of FLP; these include spectrophotometry [4], high performance liquid chromatography (HPLC) [5,6], gas chromatography-mass spectrometry (GC-MS) [7] and capillary electrophoresis [8].

Trace analysis requires the design of a method with high selectivity and sensitivity. A flourimetric method with very low detection limits and selectivity would be a suitable technique for trace analysis. Fluorescence has two wavelengths (the excitation and emission wavelength); so the two wavelengths can be controlled to increase the degree of selectivity.

However, some of the drugs are only weakly fluorescent in nature, the fluorimetric method is not of use in detecting them. On the other hand, the profen family has a carboxylic group which is known to form complexes with many metal ions that can form fluorescent products when linked to fluorogenic ligands. The use of lanthanide sensitization by chelate formation is one such approach which can be employed to determine the concentration of the organic ligand.

The efficient intermolecular energy transfer process in a solution from the excited triplet state of the ligand to the emitting level of the lanthanide ion gives the lanthanide chelates unique luminescence properties, which are manifested in a narrow banded emission line that has a long decay life-time. The sensitizing ligand is referred to as sensitizing chromophere or "antenna". The decay time ranges between nanoseconds to milliseconds, with terbium and europium having the longest decay time.

Terbium-sensitized luminescence has been applied for the determination of benzoic acid and several derivatives such as anthranilic acid [9], salicylic acid [10], terephthalic acid [11], 1,4-diamino benzoic acid [12] and profen derivatives $[13,14]$.

The efficiency of the energy transfer depends on several factors, these being the nature of the ion, the ligand type, the ligand-ion bond, the solvent, the rigidity of the molecular structure and the difference between the excited and the ground state levels [15].

In this study we report a method for the assay of FLP in pharmaceutical formulation. The method is based on the luminescence enhancement and sensitization of terbium ions upon complexation with FLP. The photosensitization was first carried out in organic solvents, and the effect of the type of solvent on the sensitization was thoroughly investigated. The effect of the concentration of metal to ligand and the stochiometry of the complex was also determined in the optimum solvent.

Furthermore, we studied the luminescence of a the FLP-Tb system in aqueous media. In order to apply the method developed in an aqueous medium, factors affecting sensitization such as $\mathrm{pH}$, surfactant type and concentration, the ligand type and concentration, and different common interferences were investigated. Using the optimized conditions established, a method was then designed and employed to determine the amounts of FLP in pharmaceutical samples.

\section{Experimental}

\subsection{Reagents}

Flubiprofen (FLP) was obtained from Sigma (St. Louis, Mo, USA). Methanol HPLC grade was obtained from Sigma-Aldrich (Germany). Terbium(III) chloride hexahydrate, 4,7-diphenyl-1,10-phenanthroline (97\%) (DPP), trioctylphosphine oxide (TOPO), ethylenediaminetetraacetic acid (EDTA), 1,10-phenanthroline and tris(hydroxymethyl) methylamine (TRIS) were obtained from Aldrich (Germany). Tween-20 was obtained from Kanto Chemical CO, INC. (Tokyo, Japan). Calcium chloride, sodium chloride, magnesium chloride, zinc chloride, ferric chloride, potassium chloride and urea were obtained from BDH Chemicals Ltd (Pool, England). FLP tablets (50 mg) were collected from the market; all other reagents and solvents were used without further purification. Ultra pure water (Milli-Q, corporation) was used for the preparation of reagents.

\subsection{Apparatus}

Luminescence measurements were performed using a Perkin Elmer LS-55 luminescence spectrometer (Perkin Elmer, Beaconsfield, UK) equipped with a xenon discharge flash lamp. The operation and data processing were controlled by the fluorescence FLwinlab Software. The excitation and emission wavelengths were set at $250 \mathrm{~nm}$ and 
SALMA AL-KINDY ET AL

$549 \mathrm{~nm}$ respectively (in the methanol system) and $285 \mathrm{~nm}$ and $552 \mathrm{~nm}$ respectively (in the aqueous system). A gate time of $1.00 \mathrm{~ms}$ and a delay time of $0.04 \mathrm{~ms}$ were used throughout. Absorption spectra were recorded on a Varian CARY 50 Conc UV visible spectrophotometer (Connecticut, USA) with $1 \mathrm{~cm}$ matched quartz cell. Measurement of pH was made with a WTW $320 \mathrm{pH}$ meter (Germany) using a combined electrode.

\subsection{Preparation of standard solutions}

FLP stock solution $\left(1.00 \times 10^{-2} \mathrm{~mol} \mathrm{~L}^{-1}\right)$ was prepared by dissolving $24.4 \mathrm{mg}$ of FLP in $10.00 \mathrm{~mL}$ of methanol. A working standard solution $\left(1.00 \times 10^{-3} \mathrm{~mol} \mathrm{~L}^{-1}\right)$ was freshly prepared daily by appropriate dilution of the stock with deionized water. Terbium(III) chloride hexahydrate stock solution $\left(0.010 \mathrm{~mol} \mathrm{~L}^{-1}\right)$ was prepared by dissolving $37.3 \mathrm{mg}$ of $\mathrm{Tb}^{3+}$ in $10.00 \mathrm{~mL}$ of distilled water. Working solutions were freshly prepared by appropriate dilution with distilled water.

Tris buffer solution $\left(0.10 \mathrm{~mol} \mathrm{~L}^{-1}\right)$ was prepared by dissolving $6.055 \mathrm{~g}$ of TRIS in $500 \mathrm{~mL}$ distilled water and the $\mathrm{pH}$ was adjusted by adding hydrochloric acid to the value of 8.00 .

Stock solutions of various surfactants were prepared by dissolving $1.00 \mathrm{~g}$ each of the surfactant in $100 \mathrm{~mL}$ deionized water in a volumetric flask. The surfactants used in this study included Tween-20, Tween-80, Triton X-100, sodium dodecylbenzene sulfonate (SDBS) and sodium dodecyl sulphate (SDS).

DPP, TOPO, EDTA and phenanthroline of concentration $1.0 \times 10^{-2} \mathrm{~mol} \mathrm{~L}^{-1}$ were prepared by dissolving $33.2 \mathrm{mg}$, $38.6 \mathrm{mg}, 29.2 \mathrm{mg}$ and $18.0 \mathrm{mg}$ of the reagents respectively in $10 \mathrm{~mL}$ methanol, and working solutions were freshly prepared by appropriate dilution with distilled water.

A $1000 \mu \mathrm{g} \mathrm{mL}^{-1}$ of the cation of each solution of sodium chloride, calcium chloride, magnesium chloride hexahydrate, zinc chloride, iron chloride, potassium chloride and urea was prepared in a $25 \mathrm{ml}$ volumetric flask.

\subsection{Preparation of drug samples}

The pharmaceutical sample was prepared by weighing FLP tablets $(50 \mathrm{mg})$. Eight tablets were separately weighed and ground homogeneously. A portion of the powder equivalent to one tablet was accurately weighed and then sonicated with methanol for $30 \mathrm{~min}$, filtered into a volumetric flask and completed to the mark with methanol to make a stock solution. A working solution of $500 \mu \mathrm{g} \mathrm{mL} \mathrm{mL}^{-1}$ was prepared in methanol in a $100 \mathrm{~mL}$ volumetric flask. Appropriate aliquots from the working solution were taken for the determination of FLP in samples.

\subsection{Preparation of water samples}

Tap water samples were filtered and spiked with convenient amounts of FLP standard solution. The final FLP concentration was in the range of $200-1000 \mathrm{ng} \mathrm{mL}^{-1}$.

\subsection{Procedure}

Aliquots of the working solution were pipetted into $10 \mathrm{~mL}$ calibrated flasks. $1.0 \mathrm{~mL}$ of $\mathrm{Tb}^{3+}, 1 \mathrm{~mL}$ of DPP solution and $0.3 \mathrm{~mL}$ of Tween-20 solution were added. The solution was diluted to the mark with $0.10 \mathrm{M}$ TRIS solution. The $\mathrm{pH}$ of the TRIS solution was adjusted to 8 . The FLP concentration range was $0-1000 \mathrm{ng}^{-1}$. The luminescence was measured at $552 \mathrm{~nm}$, using an excitation wavelength of $285 \mathrm{~nm}$. The concentration of FLP in the sample was determined from a calibration curve prepared under identical conditions.

\section{Results and Discussion}

\subsection{Spectral Characteristics}

The UV-visible absorption spectra of the FLP and Tb complex were measured in methanol and are shown in Figure 1. The absorption spectra of the FLP and FLP-Tb complex exhibited absorption in the region $210-280 \mathrm{~nm}$. However, a slight increase in the absorptivity was observed for the complex when compared to the ligand alone, especially at longer wavelengths accompanied by a slight blue shift of the wavelength of maximum absorbance indicating complex formation.

The fluorescence emission of the FLP solution $\left(1 \times 10^{-6} \mathrm{~mol} \mathrm{~L}^{-1}\right)$ in methanol was measured. A moderate fluorescence signal was observed for FLP at $\lambda_{\text {em }} 350 \mathrm{~nm}$ when excited at $251 \mathrm{~nm}$. The fluorescence signal in the shorter wavelength region has a limited analytical usefulness as it may be subjected to the interference from the matrix especially for the determination of the drug in biological samples. 


\section{A TERBIUM SENSITIZED LUMINESCENCE METHOD}

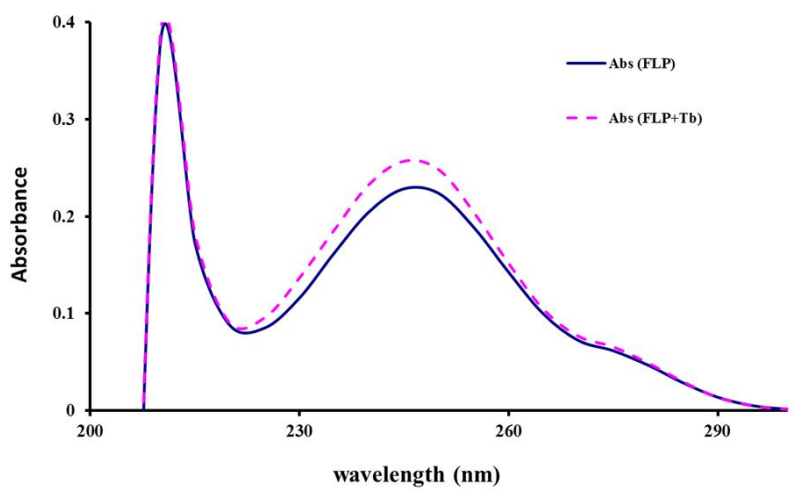

Figure 1. Absorption spectrum of FLP and FLP-Tb ${ }^{3+}$ complex in methanol. $[\mathrm{FLP}]=\left[\mathrm{Tb}^{3+}\right]=1 \times 10^{-5} \mathrm{M}$.

In order to obtain better analytical characteristics for the determination of FLP in pharmaceutical formulations, the system of FLP-Tb in methanol was investigated where FLP was used to sensitize the luminescence of the terbium ion as shown in Figure 2.

A weak time resolved signal was observed for the $\mathrm{Tb}^{3+}$; the ligand on the other hand exhibited no native time resolved emission. However, on adding FLP to $\mathrm{Tb}^{3+}$, the well known structured emission spectrum of $\mathrm{Tb}^{3+}$ was obtained (Figure 2).

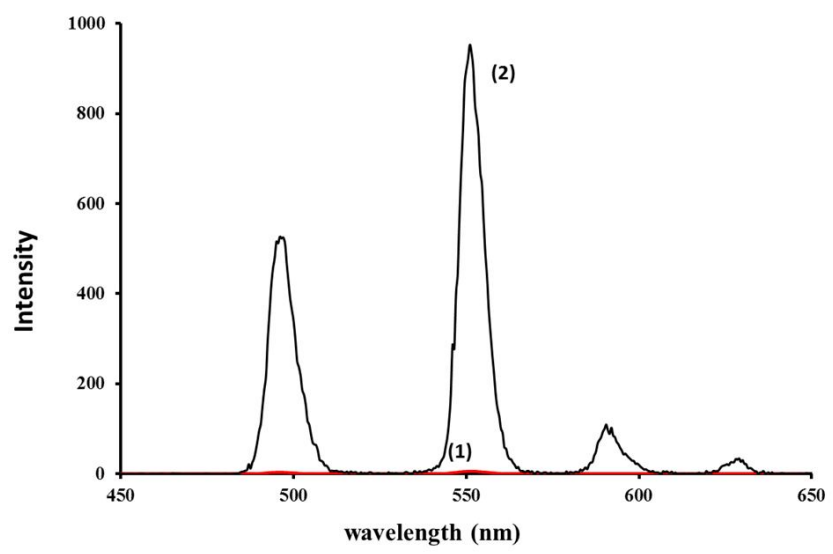

Figure 2. Luminescence emission spectra of: (1) $\mathrm{Tb}^{3+}$ in methanol. (2) $\mathrm{Tb}^{3+}$-FLP in methanol $\left[\mathrm{Tb}^{3+}\right]=1 \times 10^{-5} \mathrm{M}, \lambda_{\mathrm{em}}=549 \mathrm{~nm}, \lambda_{\mathrm{ex}}=$ $251 \mathrm{~nm}$.

The fluorescence enhancement of the $\mathrm{Tb}^{3+}$ ion in the presence of FLP and the shift in the wavelength suggests complex formation between the drug and the terbium ions. Similar shifts have been reported previously for the complexation of $\mathrm{Tb}^{3+}$ with other arylpropanoic acid derivatives [13,14].

The luminescence enhancement of the $\mathrm{Tb}^{3+}$ ion upon complexation with FLP is attributed to the efficient transfer of excitation energy from the FLP moiety to the encapsulated $\mathrm{Tb}^{3+}$ ion which can overcome the low ion extinction coefficient. For the sensitization process to be successful, the sensitizing moiety should be excited in the near ultraviolet region since the triplet energy state should have sufficient energy to provide efficient energy transfer to the ion. This excitation is well satisfied by the FLP-Tb complex which exhibits absorption at $251 \mathrm{~nm}$ in methanol. It has been reported that the longest excited wavelength of $\mathrm{Tb}^{3+}$ is at $341 \mathrm{~nm}$ [16]. The luminescence emission spectrum of the FLP-Tb complex when excited by the $\pi-\pi^{*}$ absorption band at $251 \mathrm{~nm}$ revealed the well-known bands of terbium, based on the ${ }^{5} \mathrm{D}_{4^{-}}{ }^{7} \mathrm{~F}_{6}(490 \mathrm{~nm}),{ }^{5} \mathrm{D}_{4^{-}}{ }^{7} \mathrm{~F}_{5}(550 \mathrm{~nm}),{ }^{5} \mathrm{D}_{4^{-}}{ }^{7} \mathrm{~F}_{4}(590 \mathrm{~nm})$ and ${ }^{5} \mathrm{D}_{4^{-}}{ }^{7} \mathrm{~F}_{3}(620 \mathrm{~nm})$ and they can be seen as peaks in Figure 2. The most intense peak is at a wavelength of $550 \mathrm{~nm}$ which indicates the ${ }^{5} \mathrm{D}_{4^{-}}{ }^{7} \mathrm{~F}_{5}$ transition, so it is more convenient to base the study on this peak. Although this emission band overlaps the second order scatter from the excitation wavelength, the use of time resolved mode eliminates the scattering effect as these systems have a large 


\section{SALMA AL-KINDY ET AL}

luminescence life time. Furthermore, a Stoke shift $>250 \mathrm{~nm}$ was observed indicating that there is no overlap between the $\mathrm{Tb}^{3+}$ emission band with the antenna chromophore absorption band. The close resemblance of absorption spectrum of FLP $(245 \mathrm{~nm})$ and the excitation spectrum of the FLP-Tb $(251 \mathrm{~nm})$ indicates that the excitation of the antenna chromophore may be the only photophysical pathway leading to the observable luminescence in the system [17]. The hypersensitive emission line of $\mathrm{Tb}^{3+}$ at $551 \mathrm{~nm}$ requires the optimization of different factors that affect the coordination environment.

\subsection{Complex Stoichiometry}

The optimal molar ratio of FLP:Tb ${ }^{3+}$ was determined by measuring the luminescence intensity of various mol ratios of FLP: $\mathrm{Tb}^{3+}$. The ratio of metal to ligand that will give a maximum complex was determined by using two different methods, the molar ratio method and the mole fraction method (Job's plot). In the molar ratio method, the concentration of FLP was maintained constant at $1 \times 10^{-5} \mathrm{~mol} \mathrm{~L}^{-1}$ while the concentrations of $\mathrm{Tb}^{3+}$ were varied, ranging from $0.5 \times 10^{-5}$ to $9 \times 10^{-5} \mathrm{~mol} \mathrm{~L}^{-1}$. The luminescence intensity increased with an increase in the concentration of $\mathrm{Tb}^{3+}$ till it reached a stable value. Upon extrapolating the linear part of the curve it intersects at a ratio of 4:1, which suggests a stoichiometry of $4: 1$. Therefore the concentration of terbium was maintained at a four fold excess of FLP.

The stochiometry of the complex was confirmed further by using Job's plot of continuous variation as demonstrated in Figure 3. The experimental results also indicated that the maximum fluorescence intensity was obtained at a mole fraction of 4:1.

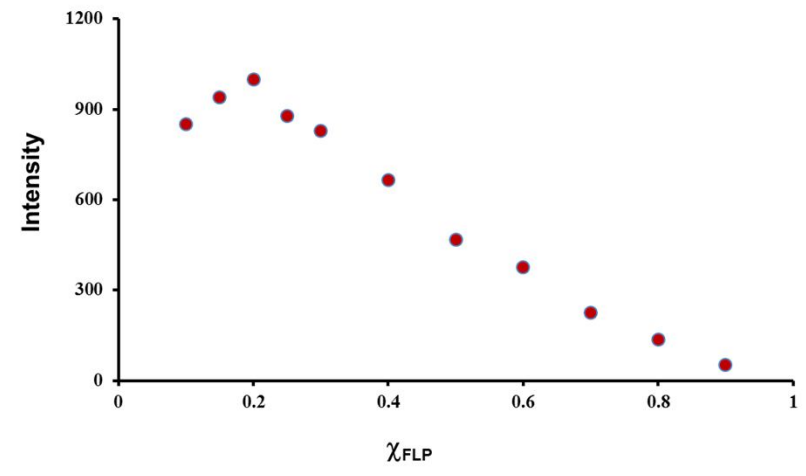

Figure 3. Job's plot of continuous variation for complexation of $\mathrm{Tb}^{3+}$ with FLP, $\lambda_{\mathrm{ex}}=265 \mathrm{~nm}, \lambda_{\mathrm{em}}=550 \mathrm{~nm}$.

The stiochiometric study was repeated many times to confirm the FLP:Tb ratio since 1:4 is an unusual result, where the common ratio of terbium-sensitized luminescence that had been reported in the literature for the determination of benzoic acid and several derivatives is 3: $1[12,13,14]$. This unusual behavior may be due to the presence of a halogen atom (F) in FLP which probably binds to the extra $\mathrm{Tb}$ atoms.

\subsection{Effect of Solvent}

Since the type of solvent used influences the luminescence intensity of the molecule, an investigation was made to study the effect of solvent on the luminescence intensity of the Tb-FLP complex. The results of the effect of solvent on the luminescence emission of the complex are shown in Figure 4. While investigating a series of alcohols, the maximum enhancement was observed in methanol, which has the highest dielectric constant ( $\varepsilon_{\mathrm{o}}$ of 32.6), and the minimum intensity was observed in the presence of butanol $\left(\varepsilon_{0}=17.1\right)$ accompanied by a slight blue shift in the emission wavelength from $557 \mathrm{~nm}$ to $550 \mathrm{~nm}$. These results indicate that the solvent polarity affects the luminescent intensity of the complex. Similar results were previously reported by Dang et al.[18], Qin et al.[19] and Al-Kindy et al. [14] for terbium and europium complexes, where maximum sensitization was observed in methanol. This behavior is explained by the higher coordination between methanol and terbium [18]. Furthermore it has been reported that as the alkyl chain in the alcohol increases, the emission intensity decreases [20]. On the other hand, low sensitization was observed in water, this being due to the effect of quenching of the excited state of the lanthanide ions by interaction with high energy vibration associated with $\mathrm{O}-\mathrm{H}$ bonds. Solvent molecules usually replace coordinated water and avoid high frequency of $\mathrm{O}-\mathrm{H}$ bond vibration, thus greatly decreasing the energy loss by coordination of water molecules. This results in an increase in the emission of the lanthanide ion. 


\section{A TERBIUM SENSITIZED LUMINESCENCE METHOD}

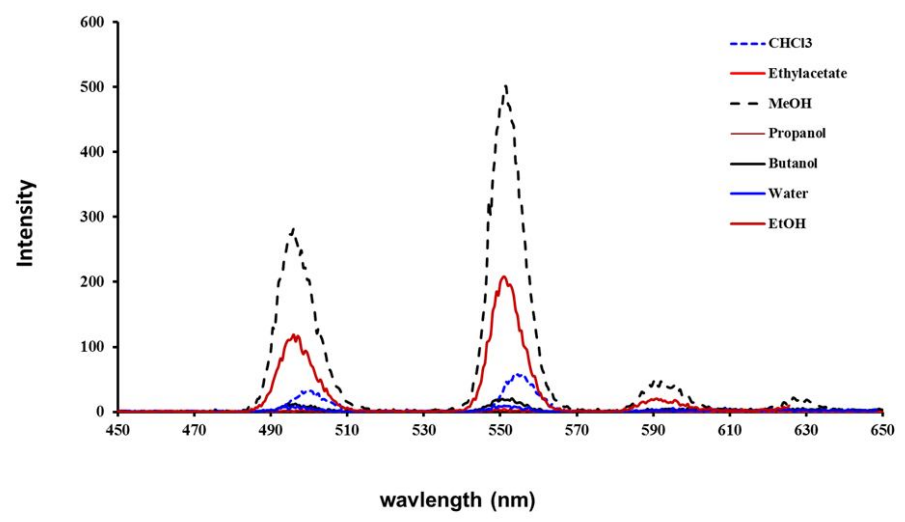

Figure 4. Effect of type of solvent on terbium sensitized luminescence emission spectra, $\left[\mathrm{Tb}^{3+}\right]=4 \times 10^{-5} \mathrm{M}[\mathrm{FLP}]=1 \times 10^{-5} \mathrm{M}, \lambda_{\mathrm{ex}}=$ $250 \mathrm{~nm}, \lambda_{\mathrm{em}}=550 \mathrm{~nm}$.

Other organic solvents such as acetonitrile, ethyl acetate and chloroform were also investigated. Acetonitrile $(\varepsilon=$ 37.5) showed extremely high sensitization where ethyl acetate $(\varepsilon=6.0)$ and chloroform $(\varepsilon=4.8)$ did not show any complexation; only the $\mathrm{Tb}^{3+}$ peaks where observed and the reason can also be referred to the solvent polarity dielectric constants $(\varepsilon)[18]$.

\subsection{Study in aqueous system}

We extended this study further to include the determination of FLP in biological samples; therefore we investigated the complexation behavior of FLP-Tb in an aqueous system. Hence factors that affect complexation such as $\mathrm{pH}$, concentration ratios of the ligand to metal, effect of surfactant and type of co-ligand were investigated. The luminescence of the Tb-FLP complex in an aqueous system revealed a band at $550 \mathrm{~nm}$ emission with an excitation at $285 \mathrm{~nm}$. These results showed a red shift in both excitation and emission wavelength when compared to the spectra in methanol, where an excitation of $250 \mathrm{~nm}$ and emission of $549 \mathrm{~nm}$ were observed. The addition of a surfactant (Tween 20) increased the luminescent intensity by the removal of the quenching effect of water, and a further enhancement was achieved when the co-ligand (DPP) was added (Figure 5).

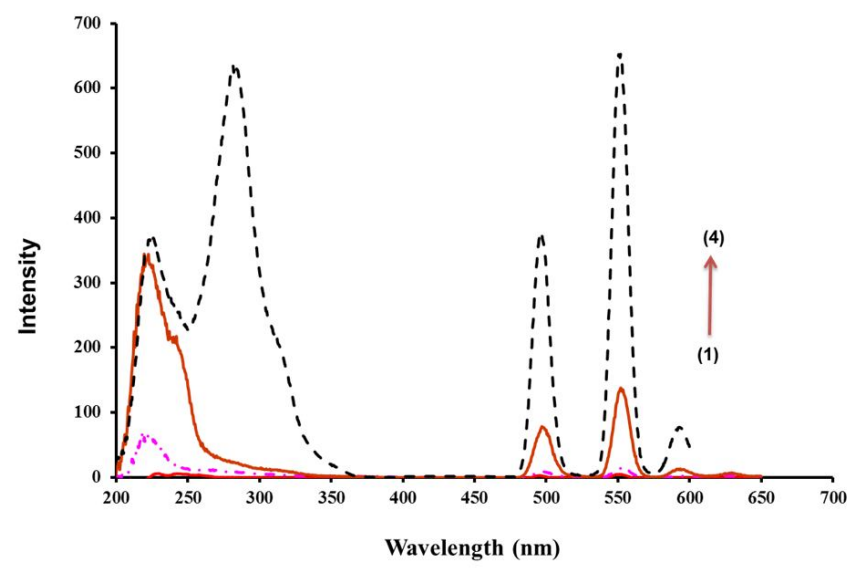

Figure 5. The effect of a co-ligand on the luminescence spectra of $\mathrm{Tb}^{3+}$-FLP system. $\left[\mathrm{Tb}^{3+}\right]=2.25 \times 10^{-6} \mathrm{M}$, $[\mathrm{FLP}] 1 \times 10^{-5} \mathrm{M}$, [coligand $]=1 \times 10^{-5} \mathrm{M},[\mathrm{Tw}-20]=0.30 \%$ (1) $\mathrm{Tb}^{3+}(2) \mathrm{Tb}^{3+}$ and FLP (3) $\mathrm{Tb}^{3+}$, FLP and Tw-20 and (4) $\mathrm{Tb}^{3+}$, FLP , Tw-20 and DPP, $\lambda_{\mathrm{ex}}=$ $251 \mathrm{~nm}, \lambda_{\mathrm{em}}=549 \mathrm{~nm}$. 
SALMA AL-KINDY ET AL

\subsection{Effect of pH}

The $\mathrm{pH}$ of the medium has a great effect on the existing form of the ligand and hence affects the complex formation. Therefore, it is necessary to determine the $\mathrm{pH}$ of the system that will give maximum complex formation. The effect of $\mathrm{pH}$ on the luminescence properties of the FLP-Tb complex was studied by measuring the luminescence of the complex in buffers of various $\mathrm{pH}$ values from 2 to 10 . An increase in the luminescence intensity was observed when the $\mathrm{pH}$ increased in the range 2 to 8.5 reaching a maximum at a $\mathrm{pH}$ of 8.0. On increasing the $\mathrm{pH}$ further, a decrease in the luminescence intensity was observed. This is a typical result, as dissociation of the carboxylic group is necessary for complexation to occur. It has been reported that the $\mathrm{pK}_{\mathrm{a}}$ of FLP is 4.2. At higher $\mathrm{pH}$, the luminescence intensity decreased due to the competitive hydrolysis of terbium forming $\mathrm{Tb}(\mathrm{OH})_{3}$ which is expected to predominate at a $\mathrm{pH}>$ 8.0. Similar results were previously reported for the complex formation between benzoic acid and terbium [12] and ibuprofen and terbium [13].

\subsection{Effect of Surfactant}

Surfactants are known to lower the surface tension of a liquid, allowing an easier spreading. Furthermore, the addition of micelles (aggregate of surfactant molecules) allows the removal of the efficient quenching effect of water in an aqueous system by forming an aggregate with the hydrophilic "head" regions in contact with surrounding solvent. As a result, the possibility of the complexation is more rapid. In addition, micelles allow the removal of the efficient quenching effect of water in the aqueous system [11]. In a previous study, it was suggested that micelles provide a discrete volume region for inclusion of the lanthanide ion and the ligand so that an energy transfer process is possible [21].

A number of surfactants were used (Tween-20, Tween-80, Triton X-100, (CTAB), (SDBS), (SDS). The concentration of surfactants was maintained at $0.1 \%$, which is above the critical micelle concentration (C.M.C.) of all of the surfactants used. Among these surfactants, Tween 20 gave the highest intensity of the luminescence signal. Since Tween-20 gave the highest enhancement of the signal, its concentration was optimized to enhance the signal even further. The luminescence intensity was observed to increase with an increase in concentration of Tween-20 from 0.1 to $0.3 \%$ and then to drop when the concentration of Tween-20 was increased. The drop in the intensity may be explained as follows; as the concentration of Tween- 20 increases the number of micelles increases until it reaches the required to solublize the whole compound. After that, the excess amount of the surfactant causes a reduction in the rate of solubilization. Therefore the concentration of Tween-20 in this study was maintained at $0.3 \%$.

\subsection{Effect of co-ligand}

One of the possible strategies to improve the luminescence properties of FLP-Tb chelates is the substitution of water molecules in the coordination sphere with synergetic agents. Several co-ligands were investigated in this study, including TOPO, EDTA, 1,10-phenanthroline and DPP with a concentration of $1 \times 10^{-5} \mathrm{~mol} \mathrm{~L}^{-1}$. The highest fluorescence sensitization was observed in the presence of DPP, the phenanthroline had the second highest intensity, and the lowest was observed in the presence of EDTA. Since DPP is insoluble in water, it was dissolved using a surfactant.

Next, the optimum concentration of DPP was investigated by monitoring the luminescence intensity of the complex in various concentrations of DPP which varied from $(0-5) \times 10^{-7} \mathrm{~mol} \mathrm{~L}^{-1}$. The luminescence intensity of DPP increased between the range $0-2.5 \times 10^{-7} \mathrm{M}$ and it reached a maximum at $2.5 \times 10^{-7} \mathrm{~mol} \mathrm{~L}^{-1 .}$ After the latter concentration, a decrease in the luminescence intensity was observed. DPP acts as a co-ligand to promote the formation of FLP-Tb complex by coordination to the terbium ion through the lone pair of its nitrogen atoms. DPP has the ability to remove water molecules from the coordination sphere. A high concentration of DPP results in competition between FLP and DPP for $\mathrm{Tb}^{3+}$ ions and hence will result in a greater enhancement of the terbium blank relative to the FLP-Tb complex.

\subsection{Concentration ratios of $\mathrm{Tb}$ in aqueous system}

Since luminescence intensity results through complex formation between terbium and FLP, it is important to recheck the maximum concentration ratio of FLP to terbium that forms the maximum complexation in an aqueous system as it was first studied only in methanol.

The optimum concentration of $\mathrm{Tb}$ (III) was obtained by varying its concentration in the range $2 \times 10^{-5}$ to $8 \times 10^{-5}$ mol L ${ }^{-1}$ while maintaining the FLP constant at $1 \times 10^{-5} \mathrm{~mol} \mathrm{~L}^{-1}$ as shown in Figure 6. From the figure it is obvious that the intensity increased with an increase in $\mathrm{Tb}^{3+}$ concentration, reaching a maximum at $4 \times 10^{-5} \mathrm{~mol} \mathrm{~L}^{-1}$, then a slight decrease in intensity was observed. This result suggests that an optimum concentration of $\mathrm{Tb}^{3+}$ is required for maximum complexation. The decrease in the intensity that was observed when adding an excess amount of $\mathrm{Tb}^{3+}$ is attributed to the decrease in the protective power of DPP, and the surfactant for FLP singlet state against radiation 


\section{A TERBIUM SENSITIZED LUMINESCENCE METHOD}

brought about collision with free $\mathrm{Tb}^{3+}$ ions. Similar behavior was observed previously for ibuprofen complexation with terbium [13].

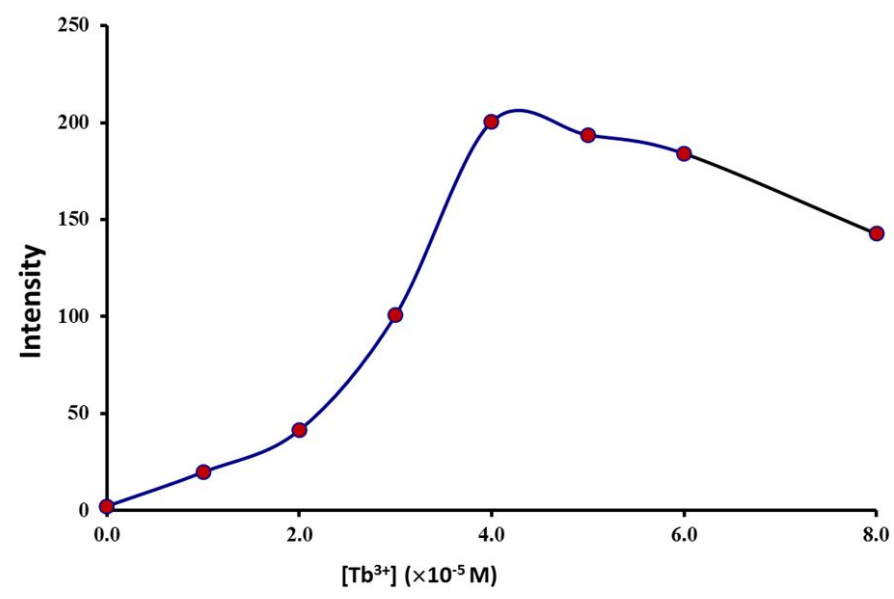

Figure 6. Influence of $\left[\mathrm{Tb}^{3+}\right]$ on sensitized luminescence. $[\mathrm{FLP}]=1 \times 10^{-5} \mathrm{M},[\mathrm{Tb}]=4 \times 10^{-5} \mathrm{M},[\mathrm{DPP}]=2.5 \times 10^{-6} \mathrm{M},[\mathrm{Tw}$ wen-20] $=$ $0.3 \%$, [TRIS $]=0.1 \mathrm{M}, \mathrm{pH}=8.0, \lambda_{\mathrm{ex}}=285 \mathrm{~nm}, \lambda_{\mathrm{em}}=552 \mathrm{~nm}$.

\subsection{Effect of Interference}

Different kinds of species are commonly present in real samples; these species are known to interfere with the complex formation. The interference can affect positively where it enhances the signal or negatively where it inhibits the signal. The effect of interference by these species was studied with a constant concentration $0.4 \mu \mathrm{g} \mathrm{mL}{ }^{-1}$ for FLP as shown in Table 1. From the table we can conclude that for most of the interfering species, the recovery percentage increases as the concentration of the substance interfering decreases. This is especially so in the case of ferric chloride, due to the paramagnetic character of the iron, which results in quenching the signal. On the other hand, the opposite occurs with zinc chloride and magnesium chloride. Both have a positive interference effect where the recovery percent increases as the concentration of the interference species increases. In order to improve the recovery of the sample, ion exchange chromatography may be used to eliminate the interference effect.

Table 1. Effect of interference on recovery of FLP.

\begin{tabular}{ccc}
\hline Interfering species & Recovery $\% \pm$ R.S.d & FLP : Interfering species \\
\hline $\mathrm{NaCl}\left(100 \mu \mathrm{g} \mathrm{mL}^{-1}\right)$ & $92.8 \pm 0.3$ & $1: 40$ \\
$\mathrm{KCl}\left(100 \mu \mathrm{g} \mathrm{mL}^{-1}\right)$ & $81.9 \pm 0.2$ & $1: 40$ \\
$\mathrm{ZnCl}_{2}\left(50 \mu \mathrm{g} \mathrm{mL}^{-1}\right)$ & $156.9 \pm 0.1$ & $1: 5$ \\
$\mathrm{MgCl}_{2}\left(75 \mu \mathrm{g} \mathrm{mL}^{-1}\right)$ & $113.9 \pm 0.8$ & $1: 20$ \\
$\mathrm{CaCl}_{2}\left(12.5 \mu \mathrm{g} \mathrm{mL}^{-1}\right)$ & $78.9 \pm 0.1$ & $1: 5$ \\
$\mathrm{FeCl}_{3}\left(2.5 \mu \mathrm{g} \mathrm{mL}^{-1}\right)$ & $19.2 \pm 0.2$ & $1: 1$ \\
$\mathrm{Urea}\left(150 \mu \mathrm{g} \mathrm{mL}^{-1}\right)$ & $92.3 \pm 0.2$ & $1: 60$ \\
\hline
\end{tabular}

\subsection{Analytical Figures of merit}

The luminescence intensity $(\boldsymbol{I})$ versus FLP concentration $(\boldsymbol{C})$ was found to be linear for a specific range. Two calibration curves were constructed, one in the organic medium (methanol) and the other in the aqueous medium. The calibration equation for the medium (methanol) was: $\boldsymbol{I}=552.71 \boldsymbol{C}+46.56$, with a correlation coefficient $\mathrm{R}^{2}$ of 0.996 


\section{SALMA AL-KINDY ET AL}

and for the aqueous medium it was: $\boldsymbol{I}=36.51 \boldsymbol{C}-0.35$, with a correlation coefficient $\mathrm{R}^{2}$ of 0.998 . The detection limit (signal to noise ratio of 3) was $10 \mathrm{ng} \mathrm{mL}^{-1}$, and the quantification limit was $33 \mathrm{ng} \mathrm{mL}^{-1}$ in aqueous media. This method offered an advantage over other luminescence methods, in that its emission is at a longer wavelength, free from interference from short-emitting species present in the matrices.

The reproducibility of the method was obtained for standard solutions of FLP and was found to be less than $2.0 \%$ $(n=7)$ showing excellent precision.

\subsection{Application}

The applicability of the method was then tested for the determination of the FLP in aqueous media. Analysis was made with pharmaceutical samples and spiked water samples. The results in Table 2 show that reasonable recoveries were obtained. These results suggest that the method is applicable to the determination of the drug in pharmaceutical samples and in water.

Table 2. Application of the proposed method to the assay of commercially available dosage forms and water samples.

\begin{tabular}{lll}
\hline Concentration of FLP claimed & FLP concentration found & \% Recovery \pm RSD \\
\hline $0.10 \mu \mathrm{g} \mathrm{mL}^{-1}$ water sample & $0.09 \mu \mathrm{g} \mathrm{mL}^{-1}$ & $95.32 \pm 1.40$ \\
$0.30 \mu \mathrm{g} \mathrm{mL}^{-1}$ & $0.26 \mu \mathrm{g} \mathrm{mL}^{-1}$ & $86.87 \pm 0.56$ \\
$0.50 \mu \mathrm{g} \mathrm{mL}^{-1}$ & $0.46 \mu \mathrm{g} \mathrm{mL}^{-1}$ & $92.41 \pm 0.62$ \\
FLP Genus $50 \mathrm{mg}$ & $49 \mathrm{mg}$ & $98.0 \pm 0.52$ \\
\hline
\end{tabular}

\section{Conclusion}

In this study it has been demonstrated that terbium-sensitized luminescence offers a simple, selective and sensitive procedure to determine FLP in pharmaceutical formulation. The enhancement of the terbium luminescence upon complexation with FLP has enabled the assay of this drug with high sensitivity and selectivity where short-lived emissions were efficiently eliminated. The method was applied to the assay of FLP in tablets and in aqueous systems with high recovery and reproducibility.

\section{References}

1. Cipriano, M., Björklund, E., Wilson, A., Congiu, C., Onnis, V. and Fowler, C.J. Inhibition of fatty acid amide hydrolase and cyclooxygenase by the $N$-(3-methylpyridin-2-yl)amide derivatives of flubiprofen and naproxen. Eur. J. Pharm., 2013, 720, 383-390.

2. Chen, Y., Sun, J., Huang, Z., Liao, H., Peng, S., Lehmann, J. and Zhang, Y. Design, synthesis and evaluation of tacrine-flubiprofen-nitrate trihybrids as novel anti-Alzheimer's disease agents. Bio. Med. Chem., 2013, 21, 2462-2470.

3. Al-Obaid, A.M. and Moian, M.S. Analytical Profiles of Drug Substances and Experiments" Vol. 22, Ed. Britair, H.G. Academic Press, New York, 1993, p431.

4. Sajeev, C., Jadhav, P.R., RaviShankar, D. and Saha, R.N. Determination of flubiprofen in pharmaceutical formulations by UV spectrophotometry and liquid chromatography. Anal. Chim. Acta, 2002, 463, 207-217.

5. Jin, Y.X., Tang, Y.H. and Zeng, S. Analysis of flubiprofen, ketoprofen and etodolac enantiomers by precolumn derivatization RP-HPLC and application to drug-protein binding in human plasma. J. Pharm. Biomed. Anal. 2008, 46, 953-958.

6. Guo C.C, Tang,Y.H., Hu, H.H, Yu, L.U., Jiang, H.H. and Zeng, S. Analysis of chiral non-steroidal antiinflammatory drugs flubiprofen, ketoprofen and etodolac binding with HAS. J. Pharm. Anal., 2011, 1, 184190.

7. Kumirska, J., Plenis, A., Łukaszewicz, P., Caban, M., Migowska,N., Białk-Bielińska, A., Czerwicka, M. and Stepnowski, P. Chemometric optimization of derivatization reactions prior to gas chromatography-mass spectrometry analysis. J. Chromatogr. A, 2013, 1296, 164-178.

8. Mei, J., Xu, J.R., Xiao, Y.R., Zhang, Q.R. and Feng, Y.Q., Immobilized phospholipid capillary electrophoresis for study of drug-membrane interactions and prediction of drug activity. Talanta, 2008, 75, 104-110. 


\section{A TERBIUM SENSITIZED LUMINESCENCE METHOD}

9. Arnoud, N. and Georges, J. Investigation of the luminescence properties of terbium-anthranilate complexes and applications to the determination of anthranilic acid derivatives in aqueous solutions. Anal. Chim. Acta, 2003, 476, 149-157.

10. Panadero, S., Gómez-Hens, A. and Pérez-Bendito, D. Kinetic determination of salicylic acid, diflunisal and their mixture based on lanthanide-sensitized luminescence. Anal. Chim. Acta, 1996, 329, 135-149.

11. Agüilar-Caballos, M.P. Gòmez-Henz, A. and Pèrez-Bendito, D. Simultaneous determination of benzoic acid and saccharin in soft drinks by using lanthanide-sensitized luminescence. Analyst, 1999, 124, 1079-1085.

12. Caro de la Torre, M.A. and Gömez-Henz, A. Evaluation of terbium(III) sensitized luminescence with benzenepolycarboxylic acids: determination of terepthalic acid in drinks samples. Anal. Chim. Acta, 2000, 407, 53-60.

13. Al-Kindy, S.M.Z. and Suliman F.E.O. Determination of ibuprofen in pharmaceutical formulations using terbium sensitized luminescence. Lumin., 2007, 22, 294-301.

14. Al-Kindy, S.M.Z., Al-Harasi Z., Suliman F.E.O., Al-Hamadi A. and Pillay A. Terbium sensitized luminescence for the determination of ketoprofen in pharmaceutical formulations. J. Fluoresc., 2009, 19, 249255.

15. Lis, S. Analytical applications of lanthanide luminescence in solution Chem.Anal. (Warsaw), 1993, 38, 443454.

16. Steemers, F.J., Verboom,W., Renihoudt, D.N., Vander, E.B. and Verhoeven, J.W. New sensitizer-modified calixarenes enabling near-UV excitation of complexed luminescent lanthanide ions. J. Am. Chem. Soc., 1995, 117, 9408-9414.

17. Werts, M.H.V., Hofstraat, J.H., Geurts, F.A.J. and Verhoeven, J.W. Fluorescein and eosin as sensitizing chromophores in near-infrared luminescent ytterbium(III), neodymium(III) and erbium(III) chelates. Chem. Phys. Lett., 1997, 276, 196-201.

18. Dang, F., Li, Y. and Liu, W. The new fluorescence enhancement system Tb- $N$-(2-pyridinyl) ketoacetamide$\mathrm{Et}_{3} \mathrm{~N}-\mathrm{Zn}$ and its application. Spectro. Chim. Acta A, 2007, 66, 676-680.

19. Qin, W., Zhang, Y., Liu, W. and Tan, M. Synthesis and infrared and fluorescence spectral properties of luminescent terbium and europium complexes with open-chain carboxylate crown ethers. Spectro. Chim. Acta A, 2003, 59, 3085-3092.

20. Ansari, A.A., Singh, N., Khan, A.F., Singh, S.P. and Iftikhar, K. Solvent effect on optical properties of hydrated lanthanide tris-acetylacetone. J. Lumin., 2007, 127, 446-452.

21. Rosen, M.J. Solubilization by solution surfactants: micellar catalysis. In Surfactants and Interfacial Phenomena, (3rd Edn.) Wiley: New York, 2004, 178-207. 